\title{
Insights into molecular properties of the human monocarboxylate transporter 8 by combining functional with structural information
}

\author{
Gunnar Kleinau1*, Ulrich Schweizer², Anita Kinne3, Josef Köhrle², Annette Grüters', Heiko Krude',
} Heike Biebermann ${ }^{1}$

\begin{abstract}
Background: The monocarboxylate transporter 8 (MCT8) is a member of the major facilitator superfamily (MFS) and transports specificly iodothyronines. MCT8 mutations are the underlying cause of a syndrome of severe Xlinked psychomotor retardation known as the Allan-Herndon-Dudley syndrome. This syndrome is characterized by abnormally high T3, low/normal T4 serum levels and slightly elevated serum TSH. To date, more than 25 pathogenic mutations in hMCT8 are known and they are valuable indicators of important regions for structural and functional MCT8 properties.

Methods: We designed a structural human MCT8 model and studied reported pathogenic missense mutations with focus on the estimation of those amino acid positions which are probably sensitive for substrate transport. Furthermore, assuming similarities between determinants of $\mathrm{T} 3$ binding observed in the published crystal structure of the thyroid hormone receptor beta occupied by its ligand T3 and the structural MCT8 model, we explore potential T3 binding sites in the MCT8 substrate channel cavity.

Results: We found that all known pathogenic missense mutations are located exclusively in the transmembrane helices and to a high degree at conserved residues among the MCT family. Furthermore, mutations either of or to prolines/glycines are located mainly at helices 9-12 and are expected to cause steric clashes or structural misfolding. In contrast, several other mutations are close to the potential substrate channel and affected amino acids are likely involved in the switching mechanism between different transporter conformations. Finally, three potential substrate binding sites are predicted for MCT8.
\end{abstract}

Conclusions: Naturally occurring mutations of MCT8 provide molecular insights into protein regions important for protein folding, substrate binding and the switching mechanism during substrate transport. Future studies guided by this information should help to clarify structure-function relationships at MCT8 which may bear broader relevance for other members of the MCT family. This includes decoding of the complete set of transport-sensitive residue positions and description of structural re-arrangements during transport.

\section{Introduction}

The Allan-Herndon-Dudley syndrome is an X-linked mental retardation first described in 1944 [1]. Neonates are lacking head-moving control and they are characterized by feeding problems. Severe psychomotor retardation becomes more obvious with increasing age and

\footnotetext{
* Correspondence: gunnar.kleinau@charite.de

'Institut für Experimentelle Pädiatrische Endokrinologie, CharitéUniversitätsmedizin Berlin, Germany

Full list of author information is available at the end of the article
}

some patients never attain speech or the ability to walk. An abnormal thyroid hormone constellation was found as a characteristic signature for this syndrome and guided the identification of mutations in MCT 8 in affected patients $[2,3]$. This constellation is a combination of high T3 levels with low/normal T4 and slightly increased TSH in human serum. To date, more than 25 pathogenic mutations have been identified including missense, nonsense, deletions, insertions, and splice site mutations [4].

\section{C) Biomed Central}


MCT8 is a member of the major facilitator superfamily (MFS) of membrane transporters and is structurally characterized by twelve transmembrane helices connected by intra- and extracellular loops. The $\mathrm{N}$ - and C-termini are both located intracellularly [5]. MCT8 was described as a specific iodothyronine transporter [6] and known substrates for human MCT8 are thyroxine (3,3',5,5'-tetraiodo-L-thyronine, T4), T3 (3,3',5-triiodo-Lthyronine), rT3 (3,3 ',5'-triiodo-L-thyronine), and 3,3'-T2 (3,3'-diiodo-L-thyronine) [6,7].

It is assumed that substrate binding by MFS transporters triggers the transition between an "outside-open" and "inside-open" conformation, the so-called "rockerswitch" model $[8,9]$. This structural movement must be linked with specific intramolecular events like rearrangement of amino acid interactions and also with substrate trans-localization. Despite the need of more supporting and detailed information for this structuralfunctional "rocker-switch" model, specific substrate-protein interactions need to be considered as to be causally related to different protein conformations. Of note, this model should be also applicable for the reverse way of substrate transport (i.e. efflux cycle) from the cytosolic to the extracellular site. So far, details and principles of the process are only partially known.

Naturally occurring missense mutations point to functionally and structurally important residues within the MCT8 protein. Mutations may affect protein folding and stability or directly modify the mechanism of iodothyronine transport [10]. We here explored the spatial localization, molecular environment and the potential function of their wild-type amino acids at a threedimensional "inside-open" MCT8 model, the only structural conformation where templates for homology models are available. We will also discuss the results of previous studies to evaluate the MCT8 homology model and to extent generally our knowledge regarding molecular mechanisms at MCTs.

\section{Material and methods}

\section{Homology modelling of MCT8}

The crystal structure of the Glycerol-3-phosphate transporter (GlpT) in an "inside-open" conformation was used as a structural template for the human MCT8 (hMCT8) homology model (PDB code 1PW4 [8]). Despite low sequence identity between this template and other members of MFS like MCT8, models based on this structural template are supported by experimental data concerning GLUT1 [11], MCT1 [12,13], and OATP1C1 [14]. The potential dimensions of MCT8 transmembrane helices (TMHs) were defined based on helices in the template crystal structure. For consistency we confirmed these dimensions with other available MFS crystal structures (PDB codes 1 PV6 and 1 PV7
[15]). For further structural information of MCTs see also the internet platform "Membrane transporter systems" at http://www.membranetransport.org.

Gaps of missing residues in loop regions of the template structure were closed either manually or by the 'Loop Search' tool implemented in Sybyl 7.35 (Tripos Inc., St. Louis, MO, USA). Side chains and loops of the homology model were subjected to conjugate gradient minimization (until converging at a termination gradient of $\left.0.05 \mathrm{kcal} /\left(\mathrm{mol}^{*} \AA\right)\right)$. The side chain orientations were refined during dynamic simulation ( $8 \mathrm{~ns}$ ) by fixing all backbone H-bonds of TMHs. This step of model generation is extended compared to the recently published MCT8 model [7] (5 ns) to get more precise potential MCT8 side chain orientations. Finally, the model was minimized without any constraints.

We have mapped naturally occurring single side chain substitutions (figure 1) on our MCT8 model (figures 2 and 3). Pathogenic deletions or insertions (reviewed in [4]) are not considered here, since they lead to incomplete proteins or they modify the particular helix-conformation and arrangements relative to each other. Therefore, such mutations are revoking a consistent structural prediction.

Structure images were produced using the PyMOL Molecular Graphics System, Version 1.3, Schrödinger, LLC.

Amino acid sequence alignment of human MCTs and the unification of residue numbering

Amino acid sequences of human MCT family members were aligned to analyze shared and divergent features in amino acid composition (figure 4). Details of the alignment procedure are recently described by Kinne and Kleinau et al. [7]. The alignment was used to highlight positions of pathogenic MCT8 mutations. This alignment enables comparison and analyses of residue conservation among human MCTs (figure 1).

We suggested previously a unifying numbering system for MCTs similar to family A GPCRs [7]. This position identifier scheme uses a highly conserved residue in each $\mathrm{TMH}$ as a common reference for all members of the MCT family. The first number is related to the particular helix, the second number after the dot determines the specific position in relation to the conserved residue. For example, the highly conserved tryptophan in TMH1 is defined as 1.50 and the highly conserved proline from $\mathrm{TMH} 2$ is defined as 2.50 (figure 4).

\section{Results and discussion}

The structural MCT8 model: molecular insights and predictivity

Structural models represent potential conformations of the target macro-molecule. Because of the well known 


\begin{tabular}{|c|c|c|c|c|}
\hline Substitution & References & $\begin{array}{c}\text { Structural } \\
\text { localization }\end{array}$ & Position & $\begin{array}{c}\text { Conservation of wild-type MCT8 } \\
\text { residues among nine hMCT } \\
\text { members } \\
\text { hMCT8/all others }\end{array}$ \\
\hline H192R & review [4] & TMH1 & 1.67 & $1 / 9$ \\
\hline S194F & {$[21,22]$} & TMH1 & 1.69 & $4 / 9$ \\
\hline G221R & {$[22,23]$} & $\mathrm{TMH} 2$ & 2.38 & $3 / 9$ \\
\hline A224V & {$[3,21]$} & TMH2 & 2.41 & $3 / 9$ \\
\hline A224T & {$[24]$} & TMH2 & 2.41 & $3 / 9$ \\
\hline V235M & {$[21,22]$} & TMH2 & 2.52 & $2 / 9$ \\
\hline $\mathrm{R} 271 \mathrm{H}$ & {$[21,24]$} & ECL2/TMH4 & 4.39 & $1 / 9$ \\
\hline G282C & review [4] & TMH4 & 4.50 & 9/9 \\
\hline $\mathrm{P} 321 \mathrm{~L}$ & [23] & TMH5 & 5.59 & $3 / 9$ \\
\hline G401R & review [4] & ICL3/TMH7 & 7.39 & $6 / 9$ \\
\hline L434W & {$[21,22]$} & ECL4/TMH8 & 8.39 & $6 / 9$ \\
\hline R445C & review [4] & TMH8 & 8.50 & $8 / 9$ (one Lys) \\
\hline D453V & review [4] & TMH8 & 8.58 & 5/9 (and 3 Asn) \\
\hline L471P & {$[3,21]$} & TMH9 & 9.49 & $2 / 9$ \\
\hline L512P & [2] & TMH10/ICL5 & 10.63 & $5 / 9$ \\
\hline P537L & [25] & TMH11 & 11.49 & 9/9 \\
\hline G558D & [26] & TMH12 & 12.54 & $7 / 9$ \\
\hline G564R & {$[27]$} & TMH12 & 12.60 & $8 / 9$ \\
\hline L568P & {$[21,22]$} & TMH12 & 12.64 & $7 / 9$ \\
\hline
\end{tabular}

Figure 1 Naturally occurring missense substitutions in human MCT8. In this table naturally occurring pathogenic amino acid substitutions known for MCT8 are summarized. Specific background-colours indicate structural/functional and biophysical specificities: violet - mutations at or to proline/glycines, respectively; red - side chain substitutions in the vicinity of the presumed substrate channel (figures 2 and 3); green - amino acids pointing towards the membrane. We also provide a summary on conservation of respective amino acids within the MCT family based on the alignment in figure 4 (bold are positions where more than half of the MCTs have an identical amino acid). The unified position numbering suggested in a recent study [7] is provided (for description see section Material and Methods).

causal dependency between specific biological functions (like substrate binding or transport mechanisms at MCTs) and particular structural features, structural models are useful to guide experimental approaches or to support the interpretation of experimental data. In our recent study, we identified two functionally important amino acids located at TMH8 and TMH10 [7]. In this study we started with the hypothesis that two specific charged amino acids in the transmembrane region $\left(\mathrm{R} 445^{8.50}\right.$ and $\left.\mathrm{D} 498^{10.49}\right)$ may interact with the amino acid backbone of iodothyronines. To prove this hypothesis, we have mutated both residues to alanine and tested transport of the natural substrate T3, as well as of two substrates lacking either the carboxyl or the

A)

B)

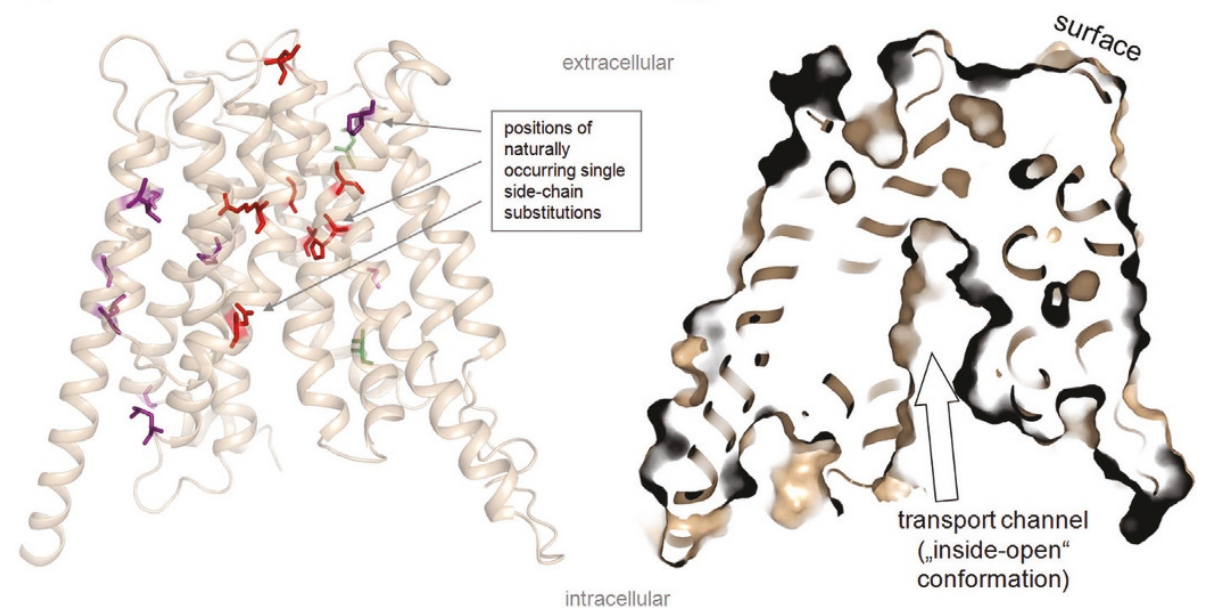

Figure 2 Homology model of human MCT8 with highlighted naturally occurring side chain substitutions. A) We highlighted wild-type amino acids of known naturally occurring mutations on the homology model of monocarboxylate transporter 8 in a conformation open to the cytosol (see figure 1). Colours were assigned according to the colour scheme in figure 1. From this three-dimensional mapping it becomes clear that none of these mutations are localized in the extra- or intracellular loops. B) The clipped surface-presentation of the MCT8 protein reveals the presumed substrate channel from the intracellular side. 
A)

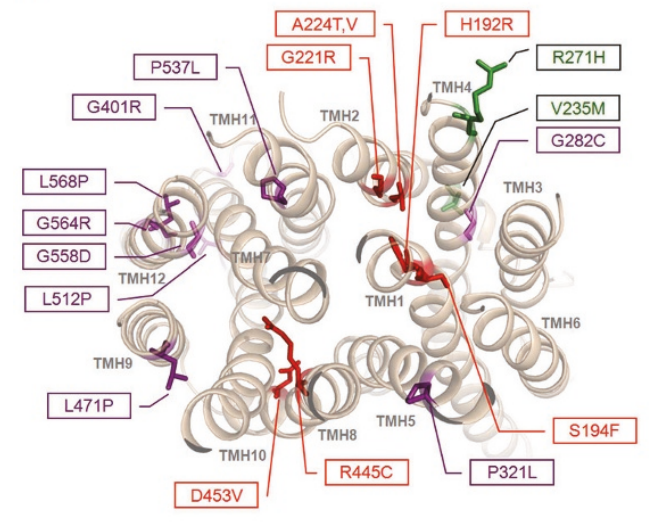

B)

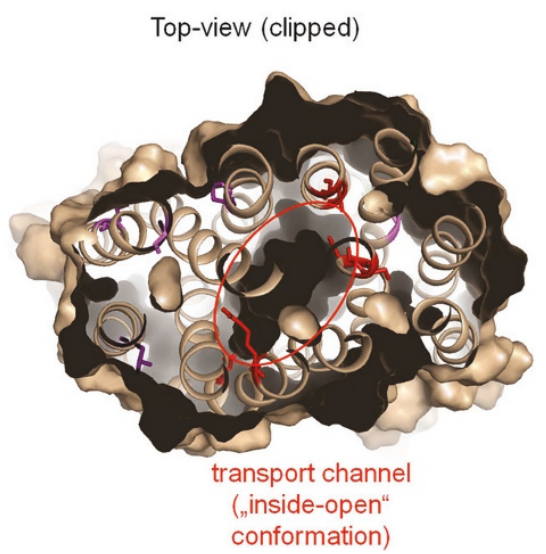

Figure 3 Homology model of the human MCT8 with mapped details of pathogenic mutations and potential localization of the transport channel switching region. A) The top-view on the MCT8 homology model with annotated pathogenic side chain substitutions reveals a detailed insight into the distribution of known mutations. The colour code given to the side chains (sticks) is in accordance with described colour scheme in figure 1. B) This clipped surface top-view visualizes the spatial relation between the wild-type amino acids of mutant positions and the "inside-open" transport channel conformation. Amino acids that are localized at the transition between the open and closed channel part are coloured red (red circle). These residues might be involved in the switching process between the "inside-open" and "outsideopen" conformations.

amino group, 3,3',5-triiodo-thyronamine and 3,3',5triiodo-thyroacetic acid. Both alanine mutants were inactive towards all three substrates. These findings support our assumption that both amino acids are important for substrate transport. The MCT8 model also predicted a potential salt bridge between both amino acids and an H-bond from R445 to the helix-backbone adjusting the relative orientation between TMH8 and TMH10. In conclusion, these two residues may have a dual function for substrate interaction and for the conformational switch mechanism. Interestingly, positively charged residues corresponding to MCT 8 position $445^{8.50}$ can be found in all MCTs. This position and spatial region may bear a fundamental role, since also in other twelve helix-transporters positively charged residues are suggested or evidenced to participate in the transport mechanisms, e.g. for GlpT [8], MCT1 [13], and OATP1C1 [14]. Finally, our findings were supported by the recent report of a naturally occurring mutation at MCT8 position 445 (R445C, reviewed in [4]).

To further evaluate the reliability of our MCT8 model, we retrospectively examined other recently published functional data. An alanine scanning study focussing on all 10 cysteines within hMCT8 [16] was performed to test the contribution to homo-oligomeric MCT8 arrangements. The alanine mutants did not alter MCT8-mediated substrate transport. We have mapped these cysteines on our MCT8 model and found that none of the cysteine side chains participates directly in the substrate channel, except C283 close to the cytosolic entry side (figure 5). Accordingly, the cysteine to alanine substitutions (shorter side chains than of cysteines) did not alter properties of the transport channel and are likely not involved in transport. Finally, seven (C184, C231, C244, C281, C283, C491, C497) out of the ten cysteines are located inside the transmembrane part. Therefore, only the extracellular 3 cysteines (C436, C481, C546) in a non-reducing environment could in principle participate in intermolecular dimer-contacts via disulfide bridges. Of note, they are not in spatial distances to each other (more than $20 \AA$ ) to form intramolecular disulfide bridges.

Naturally occurring side chain substitutions are indicators for functionally and structurally important MCT regions

We here explored the spatial localization of MCT8 mutations in the three-dimensional "inside-open" MCT8 homology model. First of all, we found by comparison of hMCT amino acid sequences and the MCT8 model that:

1. All single side chain missense mutations are located within TMHs or at helix-loop transitions (figure 2A), but not at extra- or intracellular loops. This finding highlights the transmembrane helices as most important for MCT8 function.

2. Pathogenic MCT8 mutants are localized to a high degree at conserved wild-type amino acids among MCTs (figure 4).

Conserved residues of related proteins (like the MCT family) indicate amino acids of functional and/or structural importance. Wild-type positions of MCT8 mutations at helices 5 to 12 are conserved more than $50 \%$ 

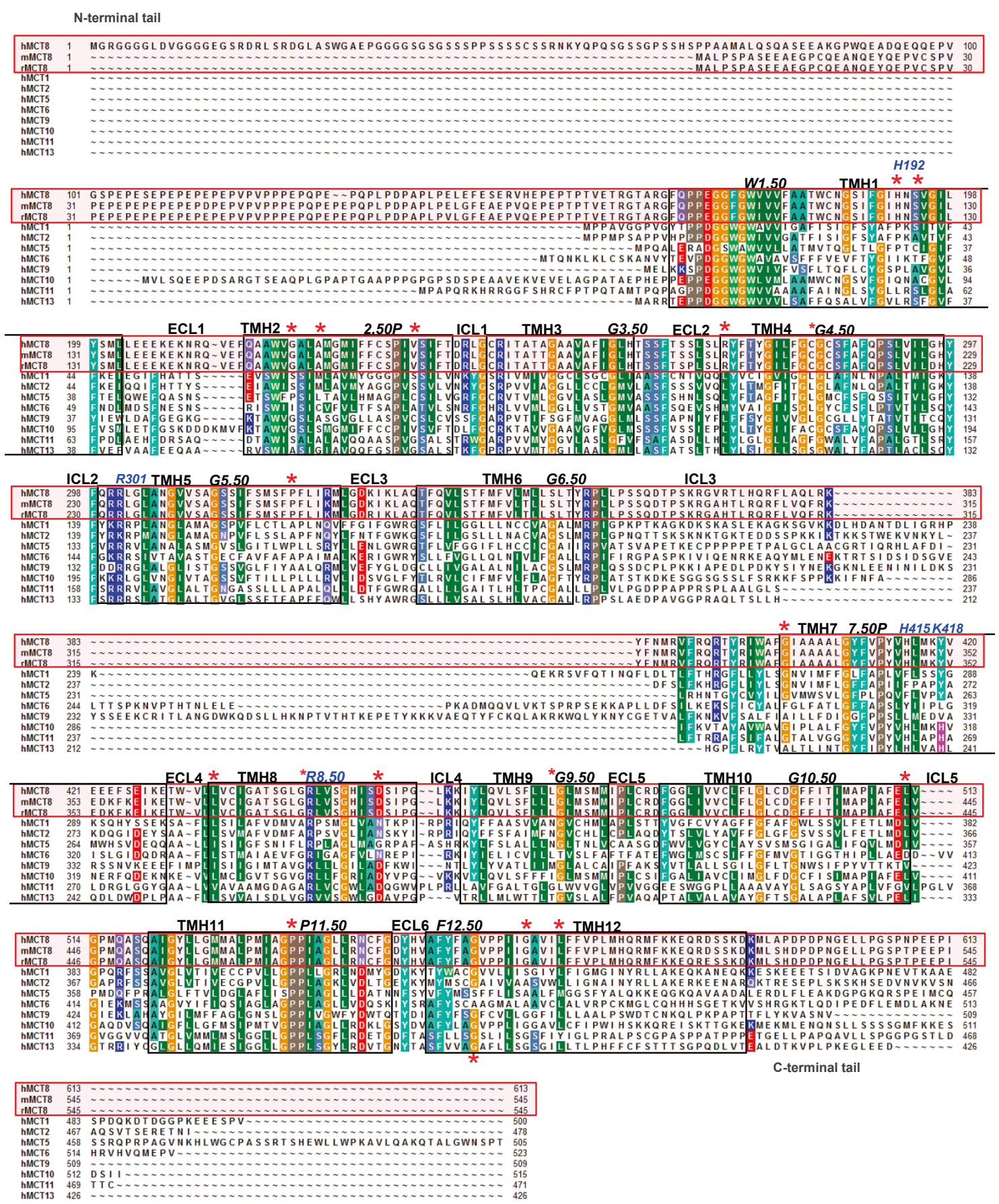

Figure 4 Alignment of the human MCTs with highlighted positions of pathogenic mutations reported for MCT8. This alignment compares the human MCT sequences and in addition MCT8 of rat and mice (MCT8 are red boxed). Conserved residues are marked by different colours according to their biophysical properties (blue - positively charged, red - negatively charged, green/orange - hydrophobic, gray hydrophilic, black - proline, cyan - aromatic). Black boxed are the potential dimensions of the transmembrane helices (TMHs) based on the X-ray structure of the E.coli Glycerol-3-phosphate transporter, another member of the major facilitator superfamily which was used as a structural template to build the MCT8 homology model. Red stars above the hMCT8 sequence indicating positions where naturally occurring mutations for the hMCT8 are reported. Few of them are at positions with a high degree of conservation like R445 (R8.50). In addition, the positively charged residues and histidines hypothesized in this study as to be potentially involved in substrate transport (figure 7) are annotated above the sequences in blue. For each helix the most highly conserved residue is numbered according to a unifying numbering system (see Material and Methods). 

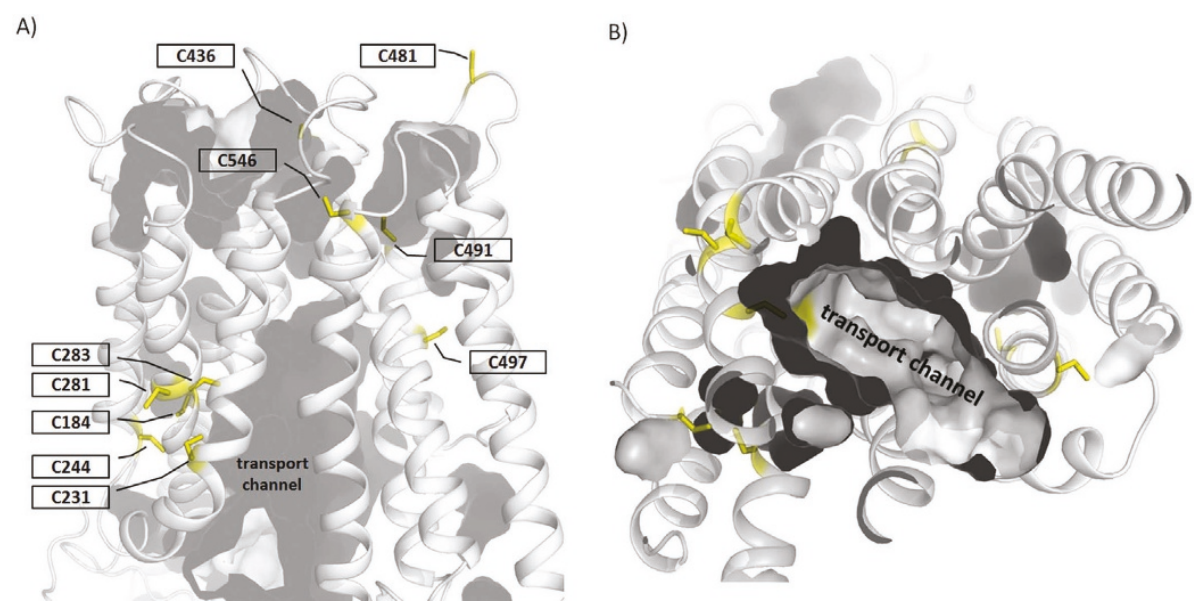

Figure 5 Distribution of cysteines in the human MCT8 homology model. To evaluate our MCT8 model on consistency we examined already published functional data. Alanine mutants of all ten cysteines in the hMCT8 did not alter MCT8 functions such as substrate transport [16]. A) With the exception of C283 at the cytosolic channel region our "inside-open" MCT8 model suggests that none of the MCT8 cysteine side chains directly participates in the substrate channel. In conclusion the experimental data and the implications from the MCT8 model are in agreement with each other. B) The cytosolic view with clipped intracellular loops shows that most of the cysteines are localized at distance from the substrate channel. (Cavities in the model are highlighted by inner surfaces.)

among hMCTs. Three out of nineteen positions are conserved to $100 \%$, four positions are conserved to $90 \%$. In conclusion, it can be speculated that mutations at highly conserved amino acids likely cause pathogenic dysfunction. These positions appear as essential for hMCTs and structural/functional similarities especially at these particular positions are preserved.
Furthermore, we have subdivided pathogenic mutations into two main groups (figure 1): I. mutations either at or to proline/glycine which leads likely to structural changes between helices or directly at helices (prolines in helices supporting kinks); II. side chain substitutions located close to the putative substrate channel which might directly modify substrate transport.
A)

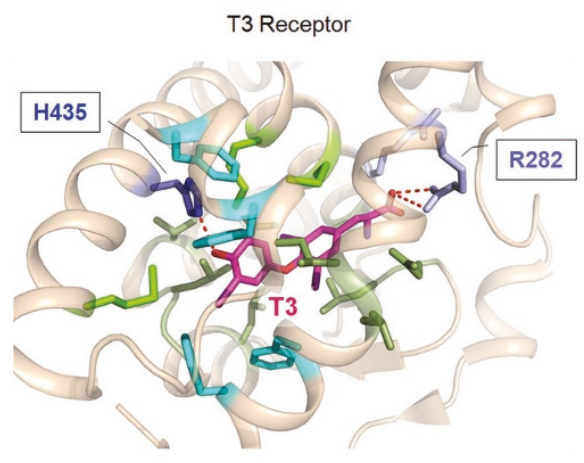

B)

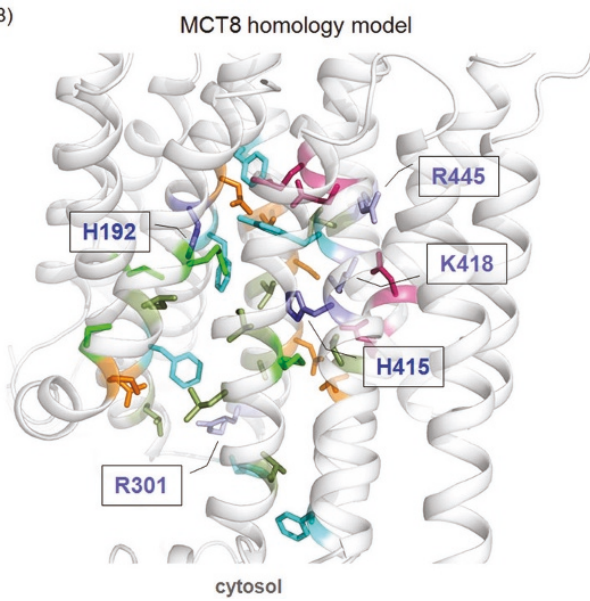

Figure 6 Details of the T3 receptor beta complex in comparison with determinants of the MCT8 transport channel. A) The crystal structure of the T3 receptor beta ligand binding domain (PDB code 3GWS [20] backbone-ribbon) reveals detailed insights into the binding mode of this hormone. Surrounded by hydrophobic (green) and aromatic (cyan) amino acids, T3 (magenta) binding is characterized by H-bonds to an arginine and a histidine. According to the assumption of analogy for T3 binding (T3 receptor, A) to different proteins, such specific complementary residues might be generally mandatory for hormone binding/transport. B) In the MCT8 homology model two histidines (H192, H415) and three positively charged residues (R445, K418, R301) are predicted to point inside the putative substrate transport channel (sticks with labels). Further amino acids in the transport channel are also highlighted by sticks: orange - hydrophilic residues without charges, magenta negatively charged residues. 

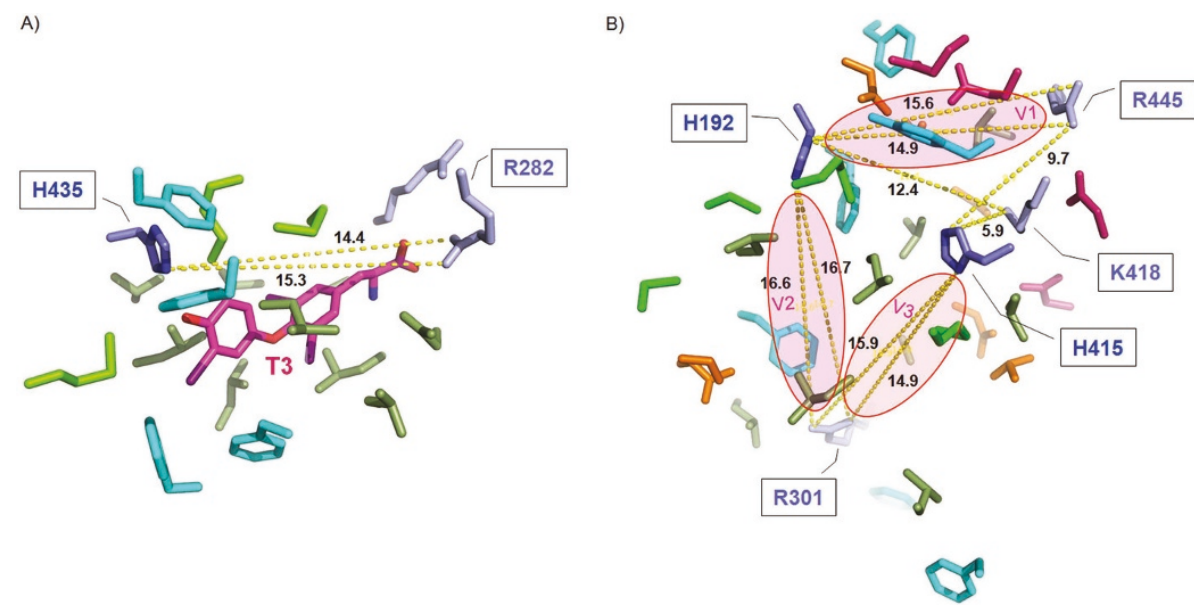

Figure 7 Applications for potential modes of T3 binding at МСТ8 by comparison between the crystallized hormone/receptor complex and the structural MCT8 model. A) T3 is bound to its receptor in a specific mode characterized by several molecular interactions like H-bonds to a positively charged arginine and a histidine (figure 6A). The amino acid side chains are in a certain distance to each other in a range between 14.4 and $15.3 \AA$. B) Measurement of the distances between the histidines and positively charges residues in the MCT8 model ("insideopen" conformation) reveals three combinations of Arg-His pairings in a similar distance to each other (red translucent regions V1-V3 (V-Variant)). Although the distance between R445 (highly conserved among the MCTs) and H192 is around $15 \AA$, some hydrophilic amino acids are in close spatial distance (V1) which would cause sterical clashes with the T3 substrate. In another transporter conformation this might be different. Of note, R445 and H192 are functionally important as demonstrated by pathogenic mutations (figure 3A). The importance of R445 for transport was also shown by directed mutagenesis.

\section{Mutations at or to prolines and glycines, respectively, cause structural misfolding}

Interestingly, for MCT8 the majority (7 out of 9) of proline/glycine mutations (P/G-X, e.g. P537L) or mutants to proline (X-P, e.g. L512P) are located at helices 9-12 (figure 1, figure 3A). They involve conserved amino acids among the MCTs. Prolines and glycines are known to support kinks and bulges in helices [17-19]. This has strong impact on both the helix (local) and the protein (global) structure. For that reason structural alterations of the helices by mutations at or to proline/ glycine, respectively, can be assumed to cause MCT8 misfolding by modification of helix conformation. In consequence deficiencies in cell surface expression of such mutants have been observed [10]. Additionally, for the P/G-X mutants it can be assumed that substitutions of prolines or glycines with enlarged side chains like leucine are predestined to cause sterical clashes in a tight micro-environment of interacting side chains.

\section{Pathogenic MCT8 mutations indicate important determinants and regions for substrate transport}

Wild-type amino acids of those mutants that are close to the transport channel (figures $2 \mathrm{~A}$ and $3 \mathrm{~B}$ ) might be directly or indirectly involved in substrate transport mechanisms. Interestingly, amino acids H192, S194, G221, A224, and R445 are located at a similar spatial layer which lies at the closed bottom substrate channel viewed from the intracellular side (figures $3 \mathrm{~B}$ and $5 \mathrm{~B}$ ).
These residues are in a specific region that must be modified or "opened" for substrate transport. Therefore, they might be involved in the switching mechanism between an "inside-open" and "outside-open" conformation. Particular residues here may function thereby as gate keepers as already described for R445 [7]. Interactions between neighbouring helices as suggested for R445 may constrain the "bottle neck like" conformation of this region. During the conformational switching such constraints must be re-arranged to enable structural movements of the helices. However, it needs more experimental characterization to clarify the detailed molecular involvement of each of these wild-type amino acids, but we can assume that the pathogenic substitutions do not maintain MCT8 transport function.

\section{Potential T3 binding sites in the MCT8 substrate transport channel}

One may assume that MCT8 substrates bind along the open channel-part in the "inside-open" MCT8 model. In order to identify amino acids which may contribute to substrate binding and transport, we used a comparative strategy. The previously published crystal structure of the T3-bound thyroid hormone receptor (TR) beta ligand binding domain (PDB code 3GWS [20]) reveals detailed insights in T3 binding (figure 6A). Surrounded by hydrophobic aliphatic and aromatic amino acids, T3 is bound by $\mathrm{H}$-bonds to an arginine and a histidine (figure $6 \mathrm{~A}$ ). Side chains of these two residues are in a 
defined spatial distance to each other of around 14-15 $\AA$ (figure 7A). Assuming a certain degree of analogy in T3 binding, such specific residues, their biochemical properties and their arrangement in the binding pocket, might represent a typical pattern for T3 binding. In the MCT8 homology model two histidines (H192 ${ }^{1.67}$, $\mathrm{H} 415^{7.53}$ ) and three positively charged residues $\left(\mathrm{R} 445^{8.50}, \mathrm{~K} 418^{8.56}, \mathrm{R} 301^{5.39}\right)$ are predicted to point towards the substrate transport channel (figure 6B). Measurement of the side chain distances between the histidines and positively charged residues ("inside-open" MCT8 conformation, figure $7 \mathrm{~B}$ ) reveals three combinations of Arg-His pairs at a similar distance to each other as in the T3-TR beta complex. These pairs and distances are shown in figure $7 \mathrm{~B}$ as potential binding regions for T3 in the channel cavity. Of note, although the distance between R445 (highly conserved among the MCTs) and H192 is around $15 \AA$ like in the T3 receptor, few other amino acids are in close spatial proximity likely would lead to sterical clashes with substrate in this conformation. In an "outside-open" conformation this might be different.

Nevertheless, R445 and H192 have already been shown to be functionally important (references in [7]). The present comparative analysis suggests two new potential key-players for substrate transport: R301 ${ }^{5.39}$ (highly conserved among the hMCTs 1-13) and H415 ${ }^{7.53}$ (conserved in hMCT8, hMCT10, hMCT11, and hMCT13). Both amino acids have not yet been investigated experimentally. R301 at TMH5 is, in contrast to the two other positively charged residues inside the channel, localized more closely to the intracellular side. Noticeably, in all three potential variants of T3 binding modes always one amino acid is linked to the potential "switching sensitive" region at the closed bottle neck of the transport channel.

\section{Conclusions}

Naturally occurring mutations in MCT8 provide molecular insights into protein regions important for protein folding and the switching mechanism during substrate transport. This was shown here by the assignment of mutations especially at TMH9 to TMH12 leading to MCT8 misfolding. Furthermore, by highlighting specific positions of pathogenic mutations in the three-dimensional structure, we have specified a critical region for the transition between the postulated "inside-open" and "outside-open" conformations. These amino acids are located at a bottle neck of the transport channel cavity, surrounded by substrate binding-sensitive amino acids. Our MCT8 model has guided several new insights in the relationship between sequence, structure and function of this transporter. This model is in accordance with experimental data and is predictive to support the identification of functional key-player amino acids. Future studies based on this model are aimed to clarify unknown details of molecular events at MCT8 and likely at other members of the MCT family. This includes: 1. identification of the complete set of transport-sensitive residues, 2 . description of structural rearrangements, 3 . identification of binding-sensitive residues, 4. details of ligand binding with molecular explanation of substrate specificity. Finally, for comprehensive understanding the relevance of oligomeric MCT8 constellations must be considered.

\section{Acknowledgement}

This work was supported by the graduate college 1208 "Hormonal Regulation of Energy Metabolism, Body Weight and Growth" (TP1 and TP3), and by the Deutsche Forschungsgemeinschaft (KL2334/2-1).

This article has been published as part of Thyroid Research Volume 4 Supplement 1, 2011: New aspects of thyroid hormone synthesis and action. The full contents of the supplement are available online at http://www. thyroidresearchjournal.com/supplements/4/S1

\section{Author details}

${ }^{1}$ Institut für Experimentelle Pädiatrische Endokrinologie, CharitéUniversitätsmedizin Berlin, Germany. ${ }^{2}$ Institut für Experimentelle Endokrinologie, Charité-Universitätsmedizin Berlin, Germany. ${ }^{3}$ Leibniz-Institut für Molekulare Pharmakologie, Berlin, Germany.

\section{Authors' contributions}

GK wrote the draft, performed and designed the studies, analyzed the data, prepared the figures; US analyzed the data, wrote the draft; AK analyzed the data; JK performed and designed the studies; AG data evaluation and discussion; HK analyzed the data, project coordination; HB analyzed the data, project coordination. All authors drafted the manuscript and approved the final manuscript.

\section{Competing interests}

There are no competing interests.

Published: 3 August 2011

\section{References}

1. Allan $W_{1}, H$ C, Dudley F: Some examples of the inheritance of mentaldeficiency:apparentlysex-linkedidiocyandmicrocephaly. Am J Ment Defic 1944, 48:325-334.

2. Dumitrescu AM, Liao XH, Best TB, Brockmann K, Refetoff S: A novel syndrome combining thyroid and neurological abnormalities is associated with mutations in a monocarboxylate transporter gene. Am J Hum Genet 2004, 74:168-175.

3. Friesema EC, Grueters A, Biebermann H, Krude H, von Moers A, Reeser M, Barrett TG, Mancilla EE, Svensson J, Kester MH, et al: Association between mutations in a thyroid hormone transporter and severe X-linked psychomotor retardation. Lancet 2004, 364:1435-1437.

4. Friesema EC, Visser WE, Visser TJ: Genetics and phenomics of thyroid hormone transport by MCT8. Mol Cell Endocrinol 2010, 322:107-113.

5. Friesema EC, Jansen J, Milici C, Visser TJ: Thyroid hormone transporters. Vitam Horm 2005, 70:137-167.

6. Friesema EC, Ganguly S, Abdalla A, Manning Fox JE, Halestrap AP, Visser TJ: Identification of monocarboxylate transporter 8 as a specific thyroid hormone transporter. J Biol Chem 2003, 278:40128-40135.

7. Kinne A, Kleinau G, Hoefig CS, Gruters A, Kohrle J, Krause G, Schweizer U: Essential molecular determinants for thyroid hormone transport and first structural implications for monocarboxylate transporter 8. J Biol Chem 2010, 285:28054-28063.

8. Huang $Y$, Lemieux MJ, Song J, Auer M, Wang DN: Structure and mechanism of the glycerol-3-phosphate transporter from Escherichia coli. Science 2003, 301:616-620. 
9. Yin $Y$, He X, Szewczyk P, Nguyen T, Chang G: Structure of the multidrug transporter EmrD from Escherichia coli. Science 2006, 312:741-744.

10. Kinne A, Roth S, Biebermann H, Kohrle J, Gruters A, Schweizer U: Surface translocation and tri-iodothyronine uptake of mutant MCT8 proteins are cell type-dependent. J Mol Endocrinol 2009, 43:263-271.

11. Salas-Burgos A, Iserovich P, Zuniga F, Vera JC, Fischbarg J: Predicting the three-dimensional structure of the human facilitative glucose transporter glut 1 by a novel evolutionary homology strategy: insights on the molecular mechanism of substrate migration, and binding sites for glucose and inhibitory molecules. Biophys J 2004, 87:2990-2999.

12. Manoharan C, Wilson MC, Sessions RB, Halestrap AP: The role of charged residues in the transmembrane helices of monocarboxylate transporter 1 and its ancillary protein basigin in determining plasma membrane expression and catalytic activity. Mol Membr Biol 2006, 23:486-498.

13. Wilson MC, Meredith D, Bunnun C, Sessions RB, Halestrap AP: Studies on the DIDS-binding site of monocarboxylate transporter 1 suggest a homology model of the open conformation and a plausible translocation cycle. J Biol Chem 2009, 284:20011-20021.

14. Westholm DE, Marold JD, Viken KJ, Duerst AH, Anderson GW, Rumbley JN: Evidence of Evolutionary Conservation of Function between the Thyroxine Transporter Oatp1c1 and Major Facilitator Superfamily Members. Endocrinology. 2010, 151(12):5941-5951.

15. Abramson J, Smirnova I, Kasho V, Verner G, Kaback HR, Iwata S: Structure and mechanism of the lactose permease of Escherichia coli. Science 2003, 301:610-615.

16. Visser WE, Philp NJ, van Dijk TB, Klootwijk W, Friesema EC, Jansen J, Beesley PW, lanculescu AG, Visser TJ: Evidence for a homodimeric structure of human monocarboxylate transporter 8. Endocrinology 2009, 150:5163-5170.

17. Yohannan S, Yang D, Faham S, Boulting G, Whitelegge J, Bowie JU: Proline substitutions are not easily accommodated in a membrane protein. Mol Biol 2004, 341:1-6.

18. Yohannan S, Faham S, Yang D, Whitelegge JP, Bowie JU: The evolution of transmembrane helix kinks and the structural diversity of $\mathrm{G}$ proteincoupled receptors. Proc Natl Acad Sci U S A 2004, 101:959-963.

19. Ceruso MA, Weinstein $\mathrm{H}$ : Structural mimicry of proline kinks: tertiary packing interactions support local structural distortions. J Mol Biol 2002, 318:1237-1249.

20. Nascimento AS, Dias SM, Nunes FM, Aparicio R, Ambrosio AL, Bleicher L, Figueira AC, Santos MA, de Oliveira Neto M, Fischer $H$, et al: Structural rearrangements in the thyroid hormone receptor hinge domain and their putative role in the receptor function. J Mol Biol 2006, 360:586-598

21. Jansen J, Friesema EC, Kester MH, Schwartz CE, Visser TJ: Genotypephenotype relationship in patients with mutations in thyroid hormone transporter MCT8. Endocrinology 2008, 149:2184-2190.

22. Schwartz CE, May MM, Carpenter NJ, Rogers RC, Martin J, Bialer MG, Ward J, Sanabria J, Marsa S, Lewis JA, et al: Allan-Herndon-Dudley syndrome and the monocarboxylate transporter 8 (MCT8) gene. Am J Hum Genet 2005, 77:41-53.

23. Vaurs-Barriere C, Deville M, Sarret C, Giraud G, Des Portes V, Prats-Vinas JM De Michele G, Dan B, Brady AF, Boespflug-Tanguy O, Touraine R: PelizaeusMerzbacher-Like disease presentation of MCT8 mutated male subjects. Ann Neurol 2009, 65:114-118.

24. Raymond L, Whibley A, Price S, Rosser E, Rahman N, Holder S, Stewart F, RTarpey P, Futreal A, Stratton M, Gold I: Barcelona. ESHG2008 2008.

25. Papadimitriou A, Dumitrescu AM, Papavasiliou A, Fretzayas A, Nicolaidou $P$, Refetoff S: A novel monocarboxylate transporter 8 gene mutation as a cause of severe neonatal hypotonia and developmental delay. Pediatrics 2008, 121:e199-202.

26. Frints SG, Lenzner $S$, Bauters $M$, Jensen $L R$, Van Esch $H$, des Portes $V$, Moog U, Macville MV, van Roozendaal K, Schrander-Stumpel CT, et al: MCT8 mutation analysis and identification of the first female with AllanHerndon-Dudley syndrome due to loss of MCT8 expression. Eur J Hum Genet 2008, 16:1029-1037.

27. Visser WE, Jansen J, Friesema EC, Kester MH, Mancilla E, Lundgren J, van der Knaap MS, Lunsing RJ, Brouwer OF, Visser TJ: Novel pathogenic mechanism suggested by ex vivo analysis of MCT8 (SLC16A2) mutations. Hum Mutat 2009, 30:29-38
doi:10.1186/1756-6614-4-S1-S4

Cite this article as: Kleinau et al:: Insights into molecular properties of the human monocarboxylate transporter 8 by combining functional with structural information. Thyroid Research 2011 4(Suppl 1):S4

\section{Submit your next manuscript to BioMed Central and take full advantage of:}

- Convenient online submission

- Thorough peer review

- No space constraints or color figure charges

- Immediate publication on acceptance

- Inclusion in PubMed, CAS, Scopus and Google Scholar

- Research which is freely available for redistribution

Submit your manuscript at www.biomedcentral.com/submit
Biomed Central 